\title{
Las TIC en educación superior. Una experiencia de aprendizaje usando Google Sites
}

\section{ICT in higher education. A learning experience using Google Sites}

\author{
Mercedes Ahumada Torres ${ }^{1}$ \\ ${ }^{1}$ Departamento Educación, Universidad Internacional de Valencia, España (mercedes.ahumada@campusviu.es)
}

\section{RESUMEN:}

El trabajo didáctico pedagógico, a nivel universitario, que se realiza usando herramientas TIC nos invitan a tener conciencia de nuevas formas de generar conocimiento. Esta propuesta exige la implicación activa del alumnado en la construcción de conocimiento individual y colaborativo ya que entran en juego destrezas de naturaleza variada. La actual sociedad del conocimiento nos invita a cambiar las prácticas universitarias centrándonos en la persona que aprende, buscando prepararlo para un mundo en permanente cambio. Se requiere múltiples competencias, como también flexibilidad y disponibilidad, para colaborar con otros en un entorno laboral que demanda formación a lo largo de toda la vida. La investigación cualitativa se ha desarrollado desde una metodología fenomenográfica, que aborda el estudio de significados y comprensiones de las prácticas de aprendizaje alcanzadas con el diseño de un Google Sites personal, por parte de un grupo de estudiantes. Se ha usado la observación participante y entrevistas semiestructuradas para valorar productos y procesos metacognitivos. El grupo ha estado constituido por 25 estudiantes pertenecientes a la Universidad Internacional de Valencia (VIU), que han sido seleccionados de forma intencionada. Los resultados son muy interesantes por la experiencia y calidad de su productividad a la vez que valoran la herramienta como una oportunidad de aprender haciendo mediante aprendizajes significativos y transferibles.

\begin{abstract}
:
The pedagogical didactic work, at the university level, which is carried out using ICT tools, invites us to be aware of new ways of generating knowledge. This proposal requires the active involvement of students in the construction of individual and collaborative knowledge as skills of a varied nature come into play. The current knowledge society invites us to change university practices focusing on the person who learns, seeking to prepare it for a world in permanent change. It requires multiple competencies as well as flexibility and availability to collaborate with others in a work environment that demands lifelong training. Qualitative research has been developed from a phenomenological methodology, which addresses the study of meanings and understandings of learning practices achieved with the design of a personal Google Sites, by a group of students. Participant observation and semi-structured interviews have been used to assess metacognitive products and processes. The group has been constituted by 25 students belonging to the International University of Valencia (VIU), who have been selected intentionally. The results are very interesting because of the experience and quality of their productivity, at the same time that they value the tool as an opportunity to learn through meaningful and transferable learning.
\end{abstract}

KEYWORDS: LEARNING, COMPETENCES, ICT, GOOGLE SITES, PHENOMENOGRAPHY
PALABRAS CLAVE: APRENDIZAJE, COMPETENCIAS, TIC, GOOGLE SITES, FENOMENOGRAFIA




\section{INTRODUCCIÓN}

Hablar de educación universitaria en pleno siglo XXI nos posiciona ante una realidad que se mueve entre experiencias formativas de diversa naturaleza. Por un lado, tenemos la formación plenamente presencial pasando luego a una experiencia tipo blended learning o semipresencial, hasta centrarnos en la que nos ocupa en el presente artículo, que es la formación que ocurre en entornos virtuales de enseñanza y aprendizaje.

Aprender dentro de un espacio mediado por las tecnologías hace que la experiencia no solo esté impregnada de grandes oportunidades para trabajar desde un modelo más constructivista sino que también provee enormes posibilidades de acercar a los estudiantes a herramientas y aplicaciones TIC de última generación. En este sentido, trabajar como profesores en dichos ambientes exige un cambio no solo de mentalidad en la forma en que pensamos nuestra acción pedagógica, sino que también nos dirige hacia una planificación rica en experiencias desde la perspectiva de los estudiantes. La llamada es distanciarnos de las clases magistrales para adentrarnos en modelos de aprendizaje más activos, dinámicos y así proveer en nuestra práctica docente de oportunidades de calidad para todos y todas.

Sin duda, en este último escenario la presencia de espacios concebidos para la formación en línea se asienta en plataformas tecnológicas ya consolidadas y dotadas de múltiples prestaciones para que ocurra la experiencia de enseñanza y aprendizaje, con alto grado de éxito para todos los usuarios del sistema. En otras palabras, desde las instituciones hay una clara intencionalidad de planificar los procesos formativos, de forma deliberada, aportando todos los insumos técnicos y logísticos para que el encuentro entre estudiantes-conocimientos y tutores sea una oportunidad efectiva de lograr las metas académicas, asociadas a los títulos y planes de estudio comprometidos por los establecimientos de educación superior.

La globalización, por otro lado, trae consigo cambios acelerados en la forma de pensar y hacer. Una demanda esencial en los estudiantes, en los tiempos actuales, es desarrollar diversas competencias y habilidades de orden superior que les facilite, no solo integrarse a su entorno más inmediato, sino que además les permita ser parte de un mundo laboral en constante cambio en la era digital y la sociedad del conocimiento. Algunos de los ejes pedagógicos que subyacen a estas nuevas demandas se orientan hacia un aprendizaje flexible y autónomo, una construcción de conocimiento individual y colaborativo frente a una presencia y uso de herramientas y aplicaciones TIC.

En el presente estudio, de tipo cualitativo transversal, se asume la propuesta aportada por Peraya y Peltier (2012), quienes exponen que las tecnologías facilitan la enseñanza de calidad en entornos virtuales de aprendizaje en los que los alumnos pueden construir y producir conocimiento sobre dichas herramientas y aplicaciones. Ciertamente las preguntas que surgen son variadas y nos llevan a desarrollar esta experiencia:

- ¿Cuáles son los significados y valor que los estudiantes otorgan a las experiencias de construcción de conocimiento usando una tecnología como Google Sites?

- ¿Qué valoraciones otorgan a un aprendizaje activo ocurrido en un entorno personal de aprendizaje?

- ¿Qué tipo de comprensiones alcanzan los estudiantes en lo relacionado con las competencias que desarrollan en su trabajo en el Google Sites de la asignatura?

- ¿En qué medida un aprendizaje autorregulado les ayuda a mejorar el compromiso con la asignatura?

Para dar respuesta a estas cuestiones se define en primer lugar un análisis documental amplio para situar el problema, para luego dar paso al diseño metodológico e implementación de la experiencia.

Presentando finalmente resultados, conclusiones y reflexiones acerca de posibles futuros estudios de naturaleza vinculante.

\section{ANTECEDENTES Y FUNDAMENTOS TEÓRICOS}

El uso de la tecnología como apoyo y vehículo a las situaciones de enseñanza-aprendizaje, más allá de la plataforma, promueve el desarrollo y consolidación de múltiples competencias que, sin duda, son exigencias clave de la sociedad actual. Por consiguiente, el uso de las TIC en los procesos formativos en entornos universitarios ciertamente permite favorecer experiencias centradas en el estudiante, potenciando con ello la atención no solo de perfiles diversos, sino que además ofertando rutas de aprendizaje flexibles. Dicho de esta forma, las tecnologías vienen a enriquecer las experiencias de enseñanza y aprendizaje, en la medida que integran no solo el cuerpo de conocimientos a adquirir sino que además ayudan de forma clara en la interacción entre estudiantes y docentes, personalizando el aprendizaje y desarrollando el potencial en todos los estudiantes. 
Así la naturaleza de la práctica pedagógica se ve dinamizada por las bondades de los recursos y herramientas TIC (tecnologías de la información y comunicación) como indican Karsenti, Meunier, Raby y Villeneuve (2011), favoreciendo con ello espacios ricos en tecnología, donde el conocimiento y la consolidación de competencias se van materializando a través de las diversas instancias de intercambio e interactividad que se generan en el uso eficiente de las TIC. Esta nueva mirada dialógica entre enseñanza-aprendizaje-tecnología nos acerca a lo expuesto por Cobo (2016) cuando plantea la necesidad de no repetir errores pasados usando la tecnología solo para transmisión de contenidos. Muy por el contrario, la invitación es a valorar las bondades de las tecnologías más idóneas a la educación superior.

No debemos olvidar que actualmente transitamos en una sociedad del conocimiento y de la información donde día a día estamos en presencia de una cultura y economía digital de acuerdo a lo expresado por Charlier, Henry, Peraya y Gillet (2010). Esta economía hace uso intensivo de las tecnologías; por tanto, nos anima y debe alertarnos para efectuar los cambios pertinentes en los procesos de enseñanza y aprendizaje, facilitando las competencias personales y profesionales de un ciudadano del siglo XXI.

Muchas veces se parte de la premisa que incluir herramientas y aplicaciones basadas en tecnología, dentro de las experiencias en entornos de formación en línea, supone un punto de inflexión en cuanto a procesos de innovación que pueden marcar la diferencia. Esto último no siempre es así; de hecho, existen algunos autores como Endrizzi (2012), que aducen que las TIC tienen un impacto positivo en el aprendizaje de los estudiantes como en su acceso al conocimiento. Ahora bien, estas mejoras idealmente, por parte de quien aprende, se relacionan con un incremento de motivación hacia la experiencia de aprendizaje. Lo anterior se hace evidente desde las comprensiones y significados que le otorgan los estudiantes a su experiencia.

En cuanto a los profesores tutores puede hacerse presente la percepción de mayor eficacia ante las metas didácticas, como asimismo en un mayor grado de seguridad sobre su desempeño docente. En otras palabras, ante la oportunidad de trabajar con diversas herramientas TIC, se observa un cierto empoderamiento tanto de profesores como de estudiantes ante los desafíos propuestos. Pero hemos de hacernos al menos una pregunta:
¿Qué tipo de uso se hace de las diversas tecnologías disponibles en procesos formativos de naturaleza universitaria?

De acuerdo a lo aportado por Lebrun, Docq y Smidts (2008), el uso de las tecnologías se orienta hacia una reproducción de las prácticas pedagógicas más tradicionales que favorecen una enseñanza clásica centrada en los contenidos de aprendizaje. Por lo que no habría valor añadido al hacer uso de ellas. Veamos el siguiente esquema elaborado a partir de lo propuesto por Guerra, González y García (2010) (figura 1), que describe algunos usos de las TIC por parte del profesorado tutor, con diversos niveles de integración.

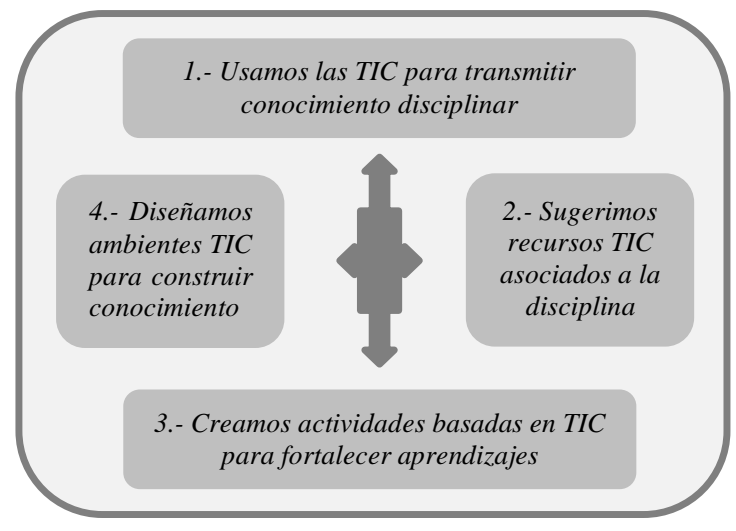

Figura 1. Tipologías de uso de TIC en la enseñanza superior. Elaboración propia.

A la luz de la figura anterior, y siguiendo a Hattie (2008), es posible afirmar que integrar las tecnologías en un entorno de aprendizaje, como es el virtual, estimula tanto la construcción de conocimiento individual como colectivo. En lo referido a las TIC, las universidades europeas y algunas latinoamericanas enfocan sus esfuerzos de integración de tecnología en la docencia mediante la recomendación de prácticas educativas que potencien interacciones de calidad entre profesores y estudiantes. Propiciando a la vez, que se provean experiencias de aprendizaje activo que ayuden en el desarrollo de competencias en los estudiantes.

Resulta interesante revisar algunos resultados a la luz de investigaciones de corte más descriptivo que nos aportan datos concretos sobre el uso de las TIC en la educación superior. La primera de ellas es la desarrollada por Castañeda, Pimienta y Jaramillo en el año 2009 en Colombia, cuyo objetivo principal era caracterizar y fortalecer la integración de las TIC en la formación inicial en dos universidades del país.

El estudio se realizó en tres fases, dentro de las cuales en la primera de ellas se aplica una encuesta a 183 profesionales universitarios para establecer el uso de las TIC por parte del profesorado en su labor docente. 
Algunos resultados, que son de interés para nuestro marco de estudio, se observan en la siguiente tabla:

Tabla 1. Uso de TIC por parte de los profesores encuestados. Adaptado de Castañeda, Pimienta y Jaramillo (2009)

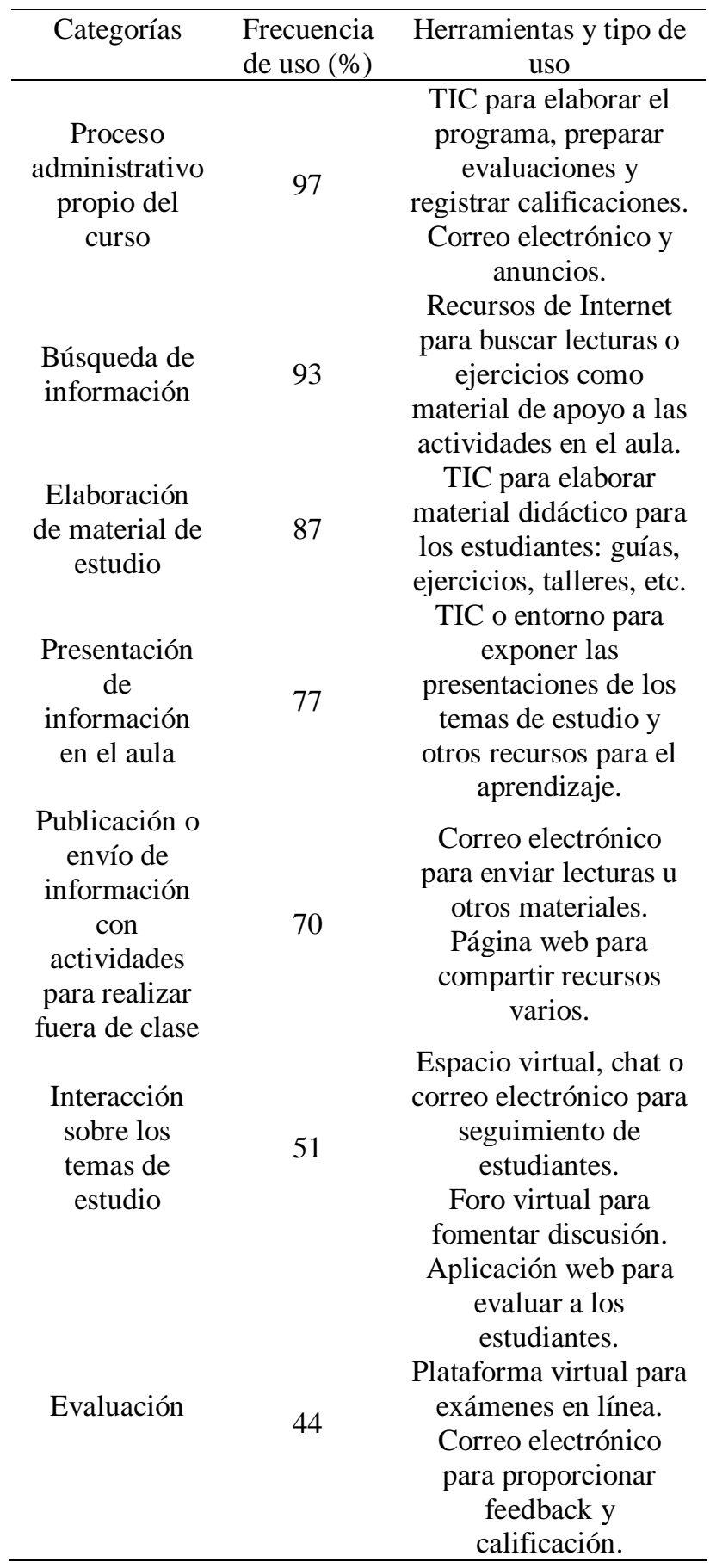

Como es posible valorar, atendiendo a la tabla 1 , buena parte de los esfuerzos docentes en la integración de TIC se dirigen a cuestiones más logísticas de funcionamiento y las que se orientan a la parte más didáctica se centran en acciones acontecidas en plataformas o sitios web complementarios a la docencia, pero con finalidades más de corte tradicional transmisivo. Por consiguiente, se puede afirmar a la luz de los datos expuestos que en esta realidad particular usar las TIC para interacciones de calidad entre docentes y estudiantes con fines de construir conocimiento son oportunidades más bien escasas.

Con relación a la presencia de las TIC en los procesos formativos en otras latitudes $\mathrm{y}$, sin perjuicio de los múltiples estudios e informes generados en este ámbito, para efectos de esta investigación abordaremos brevemente un par de cuestiones que se han delimitado por los Estados miembros de la UE en el informe Eurydice de 2011.

En el acuerdo que se concreta por parte del Consejo de la Unión Europea en el año 2009 sobre el Marco Estratégico para la Educación y Formación de 2020, se apuesta por fomentar la creatividad e innovación mediante el uso de las herramientas TIC y la formación del profesorado. Se ha podido constatar que la metodología debe estar direccionada a un enfoque centrado en el estudiante atendiendo a las directrices del aprendizaje por competencias, con un fuerte énfasis en el acompañamiento tutorial.

Ahora bien, en cuanto a las tecnologías concretamente resulta de interés destacar aquellos aspectos asociados a las competencias TIC que deben estar consolidadas durante la formación inicial en los maestros de educación primaria, entre otros profesionales.

En una era tecnológica resulta imposible que los maestros de primaria no vayan en consonancia con los perfiles asociados a tecnología que presentan sus estudiantes. Dicho de otra forma, en una sociedad donde los estudiantes son usuarios de redes sociales y abundante tecnología las TIC no pueden quedarse fuera del aula. La figura 2 presenta las principales competencias TIC:

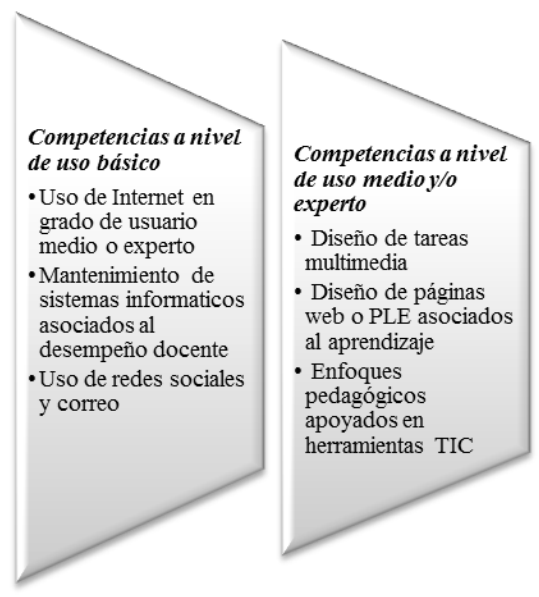

Figura 2. Algunas competencias TIC a consolidar en la formación inicial de profesorado en educación primaria 
En la actualidad un aspecto que preocupa acerca de las TIC en la formación inicial es el impacto de las tecnologías en el ámbito educativo formal. Con independencia de si estamos situados en un modelo de aprender de la tecnología, vinculado más al rendimiento académico y la satisfacción del estudiante. Igualmente desde otra mirada que es aprender con la tecnología, más orientada al desarrollo de habilidades cognitivas de alto grado de complejidad. No es lo mismo usar las tecnologías para sencillamente transmitir conocimiento que darle un uso intensivo y aplicado en la construcción de conocimiento que ayuden a resolver problemas prácticos y situados en un contexto específico.

Una última aproximación sobre el uso de las TIC en ambientes universitarios se describe en la investigación de Maaroufi (2016), que versa sobre el efecto de las TIC en las prácticas pedagógicas en una universidad marroquí. Estudio realizado en la Facultad de Ciencias de la Universidad de Oujda en la que, pasados 10 años de integración de las TIC en las experiencias formativas, se constata que los principales esfuerzos se situaron en la parte más técnico-profesional del uso de TIC que no en la formación del profesorado. Ahora bien, las buenas prácticas han emergido más a título personal y no como una política de la propia institución en la línea de una mejora sustantiva en la calidad educativa. Esto último se enmarca en la estrategia nacional de Marruecos para la integración de las TIC, donde en torno a un total de 4 áreas se ha trabajado para hacer realidad el uso de las tecnologías en las aulas universitarias, como expresa Maaroufi (2016):

- Generación de recursos y servicios digitales.

- Capacitación del profesorado en TIC para integrarlas en su docencia.

- Infraestructura TIC en la Universidad.

- Promoción de la investigación científica en la Universidad.

Los sectores privilegiados no han sido potenciados como era de esperar, ya que la Universidad ha proporcionado baja formación interna a los docentes para hacer realidad la apuesta sobre las TIC en la docencia. El seguimiento y las ayudas concretas han desaparecido en el tiempo, lo que ha bajado el grado de motivación de los profesores comprometidos. En lo referido a las prácticas pedagógicas los hallazgos globales son variados de acuerdo con la respuesta de los encuestados.

En la tabla 2 se exponen algunas constataciones atendiendo al modelo LoTI centrado en los niveles de implementación de tecnología propuesto por Moersch (1995), quien asocia el enfoque didáctico y el uso de TIC.

Tabla 2. Uso de TIC por parte de los docentes. Adaptado de Maaroufi (2016)

\begin{tabular}{cc}
\hline $\begin{array}{c}\text { Niveles } \\
\text { propuestos }\end{array}$ & $\begin{array}{c}\text { Uso de TIC por parte de los } \\
\text { docentes }\end{array}$ \\
\hline Conciencia & $\begin{array}{c}\text { Hace uso del ordenador fuera del } \\
\text { aula para fines personales. } \\
\text { Exploración } \\
\text { Las TIC se usan para complementar } \\
\text { actividades pre-diseñadas. } \\
\text { Penetración } \\
\text { Se usa la tecnología para apoyar } \\
\text { actividades de aprendizaje. } \\
\text { Las TIC se integran en el } \\
\text { aprendizaje y buscan resolver } \\
\text { problemas diversos. }\end{array}$ \\
& $\begin{array}{c}\text { Se usa la tecnología para ampliar la } \\
\text { experiencia de los estudiantes a } \\
\text { contextos reales. }\end{array}$ \\
& $\begin{array}{c}\text { Naturalidad para comprender que la } \\
\text { tecnología forma parte del }\end{array}$ \\
Refinamiento & aprendizaje activo y la construcción \\
& de conocimiento. \\
\hline
\end{tabular}

Se observan diferencias en los docentes a la hora de hacer uso de herramientas TIC que efectivamente guíen a los estudiantes hacia un aprendizaje con sentido $y$ transferible a otras situaciones o escenarios.

\section{METODOLOGÍA Y DISEÑO DE INVESTIGACIÓN}

\subsection{Metodología}

Para el objeto de investigación que nos ocupa, se ha procedido a realizar una primera fase de aproximación al problema. Para ello se ha trabajado algunos meses en una revisión minuciosa y sistemática de una gran variedad de recursos bibliográficos de naturaleza diversa para hacer emerger el estado de la cuestión sobre el uso de herramientas TIC en la educación superior. Valorando así su implicación en el fortalecimiento de competencias de diversa naturaleza en el estudiante. Lo anterior también nos ha ayudado a situar con mayor precisión nuestro objeto de estudio y las preguntas pertinentes, pudiendo definir el plan de acción hacia la experiencia tecnológica que se deseaba implementar.

En el presente estudio se abordó el objeto de investigación desde un paradigma más cualitativo, en la medida que se deseaba estudiar de forma comprensiva un aspecto de la práctica de enseñanzaaprendizaje basada en tecnologías, en su contexto 
natural. Buscando interpretar los fenómenos y prácticas desde los significados que les dan las personas implicadas, en este caso fortalecido por las instancias interactivas que se generan entre un grupo de estudiantes y la profesora que imparte la experiencia formativa asociada a la asignatura TIC y Educación. Dinamizando así la construcción de conocimiento y la transferencia del mismo a otros contextos y situaciones, ya que la experiencia es orientada al proceso ante lo cual los procesos de seguimiento tanto en la herramienta como a través de otros medios fue un valor agregado no solo para crear sinergias sino para alcanzar los objetivos propuestos.

Atendiendo a la naturaleza del objeto y las finalidades del estudio se decidió, en su momento, que lo más pertinente era aplicar una metodología fenomenográfica, de acuerdo a lo propuesto por Åkerlind (2008), con carácter emergente ya que nos facilita como investigadores ir tomando decisiones, valorando lo que va aconteciendo en el trabajo de campo. La fenomenografía se puede definir como un método de investigación que integra características del enfoque cualitativo, ya que desde su rasgo empírico lo que se desea es identificar las formas cualitativas diferentes en las que diversas personas contextualizan, perciben, experimentan y comprenden diversos fenómenos. En el ámbito educativo dicha metodología se ha ocupado fundamentalmente de la comprensión de los procesos de aprendizaje de los estudiantes, por lo que su pertinencia ante esta experiencia está bien fundamentada. Su foco investigativo aborda las diversas formas de vivir y comprender una situación o fenómeno por parte de estudiantes y profesores.

$\mathrm{Su}$ propósito, por consiguiente, es descubrir la comprensión que se alcanza acerca de fenómenos específicos. Trabaja desde el punto de vista de la concepción y comprensión de las experiencias adquiridas por parte de los agentes del acto educativo que forman parte de una experiencia innovadora, para orientar la acción a futuras mejoras de la práctica pedagógica.

Para llegar a establecer las diferencias acerca de la experiencia con la herramienta TIC por parte de los estudiantes que han participado en el estudio, se ha trabajado en base a dos tipos de análisis. Uno de ellos es el análisis referencial (González, 2010) en el cual se busca conocer y comprender qué ha supuesto esa experiencia para cada uno de los estudiantes que ha participado. Es así como se hizo un vaciado de las reflexiones metacognitivas que cada participante había incluido en su Google Sites y se aplicó 16 entrevistas semiestructuradas a los estudiantes que se situaban en diversos polos de la experiencia.

En un segundo momento se avanzó en un análisis estructural (Marton y Pang, 2005) que nos acerca a conocer cómo han adquirido el conocimiento, valiéndonos en este último aspecto de las evidencias dentro de la propia herramienta. Para ello se utilizó un levantamiento de datos verbales y escritos de las evidencias y desempeños de los estudiantes en su Google Sites.

Finalmente, se han realizado contrastes entre los hallazgos del análisis referencial con el estructural, para identificar las principales variaciones que exponen los propios estudiantes en cuanto al aprendizaje. Aprendizaje logrado a través del diseño y trabajo de construcción de conocimiento en los diversos temas de la asignatura abordados en su Google Sites personal. Esto, además, ha permitido incorporar aquello que los participantes dicen, sus experiencias, actitudes, pensamientos y reflexiones, etc., tal y como son expresadas por ellos mismos, lo que sin duda enriquece la experiencia. Aquí se ha cumplido un conjunto de características de los estudios fenomenográficos que es:

- Todos los estudiantes forman parte de un mismo grupo para la asignatura TIC y Educación, del Grado de Educación Primaria de la Universidad Internacional de Valencia (VIU).

- Se ha procedido a seleccionar informantes, es decir estudiantes, que muestran la mayor cantidad de diferencias dentro del grupo con respeto la experiencia y al objeto de estudio que se ha desarrollado.

- Ha sido posible constatar la variación dentro del mismo fenómeno desde la mirada de los participantes.

\subsection{Preguntas y objetivos del estudio}

Pregunta principal: ¿cuáles son los significados que los estudiantes otorgan a las experiencias de construcción de conocimiento usando una tecnología como Google Sites?

Otras preguntas:

- ¿Qué valoraciones otorgan a un aprendizaje activo ocurrido en un entorno personal de aprendizaje?

- ¿Qué tipo de comprensiones alcanzan los estudiantes en lo relacionado con las 
competencias que desarrollan en su trabajo en el Google Sites de la asignatura?

- ¿En qué medida un aprendizaje autorregulado les ayuda a mejorar el compromiso con la asignatura?

Objetivo general:

- Comprender y caracterizar los significados que los estudiantes otorgan a la experiencia de construcción de conocimiento usando la herramienta Google Sites en estudiantes del grado de primaria para la asignatura TIC y educación de la Universidad Internacional de Valencia.

Objetivos específicos:

- Indagar acerca de cambios en las ideas de los estudiantes sobre fenómenos específicos asociados a su aprendizaje por competencias usando herramientas TIC.

- Comprender el grado de autonomía e implicación en la construcción de evidencias de aprendizaje en un entorno diseñado y dotado de contenidos por el propio estudiante.

- Apreciar las posibles desventajas del uso de la herramienta Google Sites en el aprendizaje en plataformas virtuales.

\subsection{Muestra}

En la presente investigación, llevada a cabo en una de las asignaturas de primer semestre y curso del Grado de Educación Primaria de la VIU, en España, se ha utilizado un tipo de muestreo intencional.

Es así como inicialmente se considera el grupo completo de estudiantes matriculados en la asignatura 04GEPR "TIC y educación" con un total de 6 créditos ECTS.

La totalidad de estudiantes era 74, de los cuales 11 oportunamente han desestimado participar, pues decidieron presentar el portafolio en $2^{\mathrm{a}}$ convocatoria, lo que impedía que sus resultados se incluyesen en esta investigación. Otros, iban muy comprometidos con otros temas y expusieron su voluntad de no participar (14 estudiantes). Por diversas razones finalizaron con éxito y se mantuvieron disponibles para ser parte activa de la experiencia un total de 25 estudiantes, número interesante atendiendo a la complejidad de la tarea y el grado de dedicación que supone la amplitud del temario y las competencias en juego.
Han participado un total de 13 mujeres y 12 varones, cuyos conocimientos previos en cuanto a los contenidos, competencias y herramienta a utilizar son variados. Tenemos a casi la totalidad de estudiantes que no conocían nada acerca de la herramienta y un par de excepciones que habían trabajado con ella para fines de ocio, pero sin intencionalidad de aprender en la misma. Asumiremos que al ser esta una asignatura de 1er. curso y semestre los estudiantes tenían pocos días de iniciada su experiencia en nuestra Universidad, por lo que es doblemente valioso su implicación y calidad de las evidencias.

La experiencia de enseñanza-aprendizaje que se trabaja a través del Google Sites corresponde a la globalidad del temario de la asignatura, destacando que esta productividad forma parte de la evaluación de los aprendizajes.

\subsection{Técnicas e instrumentos}

La metodología fenomenográfica, desarrollada en esta experiencia, se fundamenta en procesos en los que las diversas interacciones y comportamientos que son observables y comprensibles para identificar significados. Incorpora lo que los participantes dicen, sus experiencias, actitudes, creencias, pensamientos y reflexiones, etc., tal como son expresadas por ellos mismos y no como uno los describe. Dichas interacciones han quedado plasmadas en la herramienta de cada participante de la experiencia y se han evidenciado en su devolución desde las evidencias de aprendizaje, que se han construido en el Google Sites y en los procesos metacognitivos.

Para obtener estos elementos a nivel de estrategias para la recolección de información destaca la observación acuciosa de los trabajos de los estudiantes y análisis de documentación complementaria aportada por ellos en la propia herramienta (Marton y Pang, 2005). Se han agregado 16 entrevistas, de tipo individual, semiestructurada en base a procesos metacognitivos sobre lo aprendido en la interactividad con la herramienta Google Sites. Se ha puesto especial atención en desvelar las acciones realizadas a lo largo de la asignatura a la hora de adquirir y construir conocimiento, buscando fortalecer competencias diversas. 


\section{RESULTADOS}

\subsection{Sobre las evidencias de aprendizaje en el sitio web del estudiante}

Durante el funcionamiento de la asignatura, que se extendió por 2 meses entre los meses de octubre y diciembre del año 2017, se procedió a trabajar desde una observación participante, facilitando no solo un seguimiento activo de los Google Sites de todos los estudiantes sino que, además, se generaron espacios de discusión en línea y por escrito dentro de la propia herramienta para ofrecer pautas de ayuda, si era solicitado por los estudiantes.

Por parte de los estudiantes se necesitó apoyos a nivel más técnico, en la medida que muchos de ellos no habían interactuado con herramientas TIC para el aprendizaje y menos con una abordada como entorno personal para aprender. Una vez acabada la asignatura a fines de 2017 se procedió a revisar, con una observación más minuciosa, los diversos Google Sites buscando caracterizar las evidencias de aprendizaje. Asimismo, se buscó una comprensión hacia la forma en que ellos construyeron conocimiento dentro los sitios web.

Entre las actividades que se propuso desarrollar en Google Sites estaban: análisis crítico sobre el temario de la asignatura, elaboración de mapas conceptuales usando herramientas en línea, creación de WebQuest para contenidos de educación primaria y blogs para fortalecer aprendizajes asociados a la asignatura.

Las principales constataciones se integran en la tabla 3 .

Tabla 3. Evidencias y comprensiones de los productos en el Google Sites de los estudiantes

\begin{tabular}{|c|c|}
\hline $\begin{array}{l}\text { Criterios } \\
\text { propuestos }\end{array}$ & Aspectos detectados \\
\hline $\begin{array}{c}\text { Calidad de las } \\
\text { evidencias de } \\
\text { los Google } \\
\text { Sites de los } \\
\text { estudiantes }\end{array}$ & $\begin{array}{l}\text { La mayoría de los } 25 \text { Google Sites } \\
\text { analizados presentan evidencias de } \\
\text { alta calidad. Los alumnos han } \\
\text { dedicado muchas horas a su trabajo } \\
\text { en esta asignatura y lo más } \\
\text { interesante es que lo han hecho } \\
\text { desde una modalidad de aprender } \\
\text { haciendo con variadas herramientas } \\
\text { TIC. } \\
\text { Existen muestras claras de } \\
\text { transferencias de la metodología } \\
\text { como de conocimientos } \\
\text { procedimentales que aplicarán para } \\
\text { otras asignaturas VIU. }\end{array}$ \\
\hline $\begin{array}{l}\text { Comprensiones } \\
\text { acerca de los } \\
\text { niveles de }\end{array}$ & $\begin{array}{l}\text { Hay una valoración positiva acerca } \\
\text { de los aprendizajes asociados al } \\
\text { temario de la asignatura, alcanzados }\end{array}$ \\
\hline
\end{tabular}

aprendizaje

logrados por

los estudiantes

\section{Implicaciones \\ del Google \\ Sites en el \\ desarrollo o \\ fortalecimiento de \\ competencias}

\subsection{Hallazgos en torno a la entrevista semiestructurada en los procesos metacognitivos}

Al momento de recibir, por parte de los estudiantes que han formado parte de este estudio, la autorización para revisar su sitio web se procedió a aplicar las entrevistas en torno a algunas preguntas de naturaleza flexible para recoger comprensiones y significados de lo experimentado a lo largo de la asignatura, en particular asociado a la experiencia de Google Sites (AG1), en términos de los procesos metacognitivos y su vinculación a construcción de conocimiento como de fortalecimiento de competencias. En este segundo momento, se trabajó desde la estrategia de entrevista semiestructurada, en la mayoría de los casos.

Otros respondieron por escrito, integrando sus percepciones y satisfacción con la experiencia. Asimismo, se ha puesto énfasis en valorar su aporte en consolidar competencias diversas y toma de conciencia por parte de los estudiantes de cómo ha ido generándose el proceso de aprendizaje. Las entrevistas asociadas a los procesos metacognitivos se leyeron tres veces en momentos diversos para luego pasar paulatinamente a los procesos de transcripción buscando aspectos iluminadores.

En esta fase lo esencial es no supeditar a los datos las categorías que pudieran haber surgido con 
antelación, sino compararlas una vez que las categorías de quien investiga hayan sido generadas. Otro aspecto clave es que el proceso de análisis es comparativo e iterativo. Esto implica que se lleva a cabo en un proceso continuo de clasificación y reordenamiento de los datos, de acuerdo por lo expuesto por Marton y Pang (2005). La idea es localizar elementos comunes y diferentes en las devoluciones de las entrevistas para luego proceder a seleccionar y organizar citas de las transcripciones.

Así llegamos al planteamiento de categorías provisionales que son valoradas y contrastadas contra los datos proporcionados hasta que el sistema de significación se consolide y sea posible hacer emerger los resultados más esenciales. uiente tabla:

Tabla 4. Aspectos referenciales y estructurales de las significaciones sobre el uso de Google Sites en la construcción de conocimiento integrando procesos metacognitivos evidenciados en las entrevistas. Adaptado de González, C. (2010)

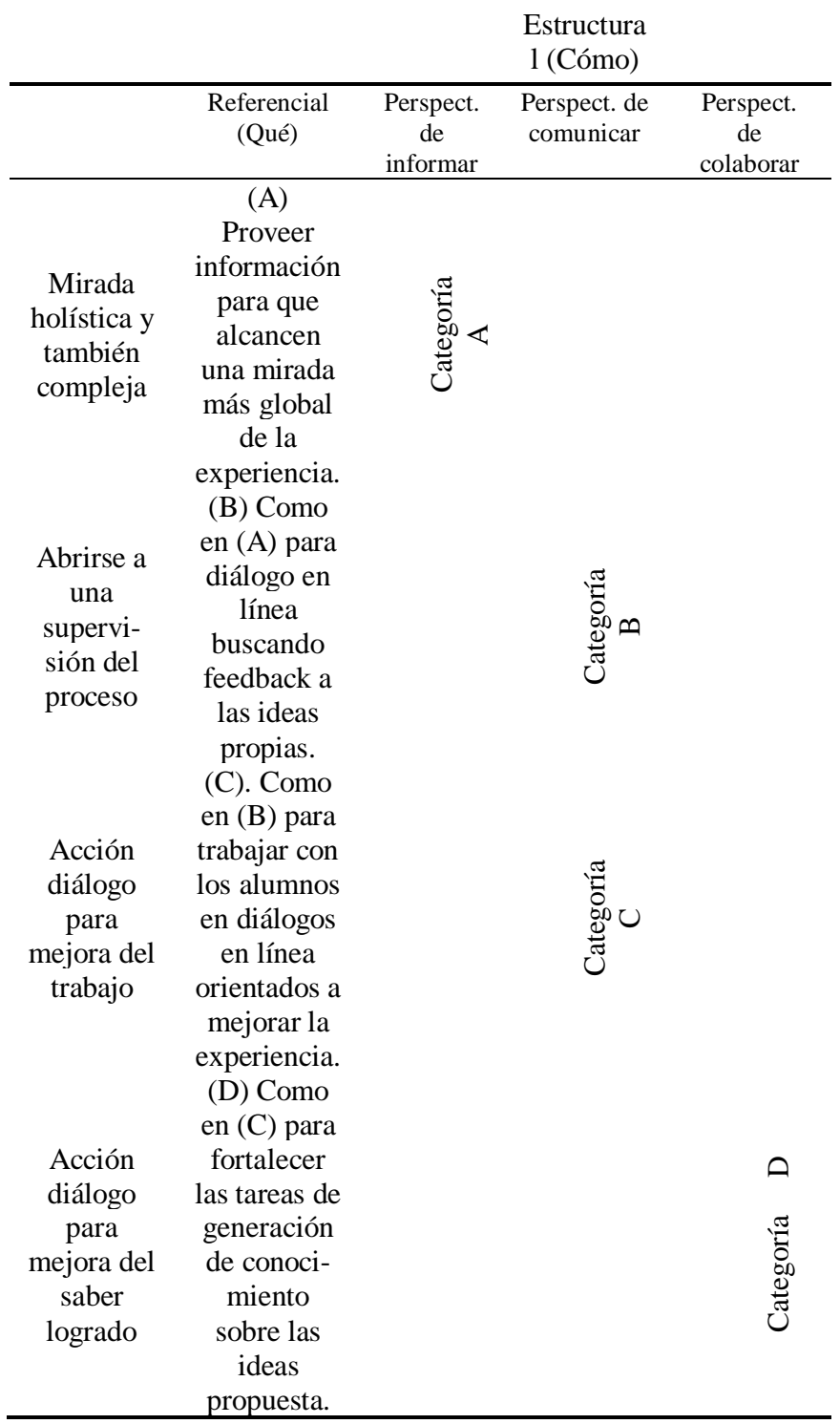

La relación entre las experiencias asumidas de forma consciente y la posibilidad de ayudar a estudiantes a pensar de forma diferente para alcanzar un grado de productividad para su experiencia en la asignatura justifica esta investigación fenomenográfica.

Si lo observamos de esta manera dialógica e iterativa de procesos y resultados, este enfoque de corte cualitativo tiene un enorme potencial para la mejora del aprendizaje y la propia práctica del profesorado.

Aquí se exponen a continuación algunas citas textuales de las significaciones metacognitivas de los estudiantes frente a la inclusión del Google Sites en la asignatura como una oportunidad de aprender haciendo y fortalecer competencias diversas.

Mantenemos la reserva de los autores pero sin duda sus aportaciones nos motivan a seguir dando lo mejor en sus experiencias de aprendizaje. A saber:

"El conocimiento sobre la propia cognición implica ser capaz de tomar conciencia del funcionamiento de nuestra manera de aprender"

"La regulación y control de las actividades que el alumno realiza durante su aprendizaje con la herramientas Google Sites es altamente significativo.

“A lo largo del proceso de creación del Google Sites me han surgido pequeños conflictos que he podido ir resolviendo por lo que he desarrollado mi habilidad de aprender a aprender"

"Se le debe dedicar horas pero con la presente actividad he conseguido implicarme con la asignatura de forma más profunda. Indagando, investigando e interesándome por muchos aspectos que hasta ahora desconocía"

"Me gustaría valorar esta experiencia hablando de mi conocimiento previo al inicio de la asignatura y, sobre todo, al inicio del trabajo con el Google Sites. Por circunstancias diferentes, soy una persona poco simpatizante con las TIC ya que no se me dan nada bien, ya sea porque en el colegio no nos enseñaron mucho o porque yo tampoco he tenido interés por aprender. Aquí he aprendido y puedo llevar a otras asignaturas mi aprendizaje"

"La cantidad de tiempo dedicada al Google Sites ha sido muy grande, enorme en comparación a las demás asignaturas del grado y considero que el esfuerzo y la inversión de tiempo valen la pena. Qué mejor manera de estudiar que utilizando las TIC: la conclusión que saco de esta asignatura es que se predica con el ejemplo"

"Respecto a cómo he aprendido, puedo decir que la base es la experimentación. Mi mejor manera de aprender y poner en práctica todos los conocimientos 
teóricos ha sido probar, equivocarme, volverlo a intentar y conseguir resultados que, finalmente, me han gustado"

"Una herramienta muy interesante para primaria y para desarrollar competencias como argumentación, búsqueda de información, entre otras"

"Lo primero que debe hacer un profesor antes de orientar a los alumnos con las TIC es aprender él mismo a utilizarlas promoviendo una clase productiva y eficiente"

"Con este Google Sites está demostrado que la utilización de estrategias educativas atractivas para el alumnado estimula a éste y consigue mayor implicación y mejores resultados en los estudios universitarios"

"Un Google Sites es una herramienta que nos permite crear conocimiento e identidad en la red"

"La construcción de este Google Sites implica planificación y estructuración clara"

"A partir del Google Sites en el aprendizaje de esta asignatura destacaría, todos los conocimientos que he adquirido, tanto de herramientas digitales como de conceptos clave"

En suma, para que las TIC efectivamente contribuyan al desarrollo de nuevas y mejores prácticas pedagógicas debe existir una convicción profunda de que es posible innovar las formas en que realizamos nuestra docencia y seguimiento.

Siguiendo a Lebrun, Docq y Smidts (2008) hemos de aplicar métodos más interactivos donde se construyan nuevos roles para los diversos actores y se genere entre ellos interacciones de calidad que efectivamente hagan emerger aprendizajes más significativos y transferibles.

\section{DISCUSIÓN Y CONCLUSIONES}

Llegados hasta aquí es interesante plantearnos algunas reflexiones desde el diálogo entre la experiencia de investigación aplicada a la práctica que se ha realizado y algunos elementos que emanan de variadas investigaciones que comparten diversos aspectos, desde la práctica realizada con la herramienta TIC como desde la mirada metodológica que se ha integrado en este estudio.

Usar las TIC en la docencia tiene un valor agregado para profesores y estudiantes. Ciertamente demanda más tiempo e implicación por ambas partes, pero los resultados son evidentes y proporcionan un plus en el aprendizaje de quien lo experimenta. Es posible valorar y concordar con autores como Dahmani y Ragni (2009), quienes plantean que el uso de herramientas tecnológicas ayuda a los estudiantes a empoderarse en el acceso y construcción del conocimiento al asumir un rol activo en su aprendizaje, en el cual las TIC tienen un papel central.

Trabajar en entornos personales de aprendizaje, como es Google Sites, permite dar espacio a pensamientos, actividades y formas divergentes de crear conocimiento, integrando el pensamiento disruptivo apoyado en tecnología. Ante los resultados y significados expuestos por los estudiantes se concuerda con autores como Arias y Cristia (2014), que postulan que una guía clara y oportuna del proceso de enseñanza-aprendizaje por parte del profesorado tutor ayuda a estimular la confianza y la creatividad de nuestros estudiantes. Aún hay mucho por hacer en esta línea ya que hemos de salir de la zona de confort para atrevernos a depositar confianza en los estudiantes.

Desde el punto de vista metodológico puede llamar la atención que se haya privilegiado la fenomenografía como parte de este estudio aplicado. Pero ello no es antojadizo, sino que corresponde a la naturaleza de las prácticas y de la experiencia en primera persona por el sujeto que aprende. No se trataba de describir de forma sencilla qué ocurre sino que se ha puesto énfasis en la forma que los estudiantes experimentan un mismo fenómeno (Garrido, 2009), siendo parte de una misma propuesta de aprendizaje facilitada por el profesor.

Las comprensiones y significados ayudan a valorar no solo los aprendizajes alcanzados, sino que permiten darnos cuenta de las propias innovaciones en la forma en que aprenden nuestros estudiantes a partir de sus decisiones y acciones.

En lo referido a las conclusiones de esta investigación, considerando los objetivos planteados, es posible confirmar desde la experiencia de los estudiantes y del propio docente lo siguiente:

- Las herramientas TIC han venido para quedarse en las prácticas de enseñanza y aprendizaje, facilitando con ello una construcción de conocimiento que parte desde un rol activo de los estudiantes.

- Un uso intensivo de la tecnología, con un buen acompañamiento tutorial, ayuda a los estudiantes a desarrollar y fortalecer competencias de diversa naturaleza.

- Trabajar con tecnología aporta a los estudiantes nuevas miradas hacia los aprendizajes que se le han planteado como 
desafío, ya que las rutas pueden ser flexibles, diversas y adaptadas por ellos mismos a sus ritmos de aprendizaje.

- Disponer de un entorno de aprendizaje apoyado en la herramienta de Google Sites ha significado un paso al frente en diversas competencias en el estudiante, lo que ha motivado un compromiso elevado con la asignatura y sus resultados académicos.

- Los grados de satisfacción con el papel de facilitador y la naturaleza del feedback por parte de la profesora tutora han sido decisivos en la oportunidad de un aprendizaje acompañado y académico con alto grado de transferencia a otros estudios y situaciones.

- Trabajar desde una mirada cualitativofenomenográfica ha resultado una experiencia interesante y gratificante, dado que esta aproximación ayuda a poner el énfasis en la mejora de la propia práctica y de las experiencias de aprendizajes de los estudiantes.

A modo de conclusión general es posible afirmar que esta experiencia nos lleva a generar nuevas preguntas y respuestas desde aspectos como la evaluación, el rol tutorial y la innovación de la práctica asociados al uso intensivo de tecnología.

\section{REFERENCIAS}

Arias, E. y Cristia, J. P. (2014). El BID y la tecnología para mejorar el aprendizaje: ¿Cómo promover programas efectivos? Banco Interamericano de Desarrollo. Recuperado http://publications.iadb.org/handle/11319/6550.

Åkerlind, G. (2008). A Phenomenographic Approach to Developing Academics' Understanding of the Nature of Teaching and Learning. Teaching in Higher Education, 13(6), 633-644.

Castañeda, C., Pimienta, M. y Jaramillo, P. (2009). Uso de TIC en la educación superior. Recuperado de:

http://www.ufrgs.br/niee/eventos/RIBIE/2008/pdf/uso_ti c_educ_superios.pdf

Cobo, C. (2016). La Innovación Pendiente. Reflexiones (y Provocaciones) sobre educación, tecnología y conocimiento. Colección Fundación Ceibal/Debate: Montevideo.

Charlier, B., Henry, F., Peraya, D. y Gillet, D. (2010). From personal environment to Personal Learning Environment. En Fifth European Conference on Technology Enhanced Learning (EC-TEL), Workshop on Mash-Up Personal Learning Environment (MUPPLE) Sept 28-Oct 1, Barcelona, Spain.
Endrizzi L., (2012). Les technologies numériques dans l'enseignement supérieur, entre défis et opportunités, Dossier d'actualité Veille et Analyses, 78.

EURYDICE (2011). Cifras clave sobre el uso de las TIC para el aprendizaje y la innovación en los centros escolares de Europa. Bruselas: Comisión Europea.

Garrido, J. M. (2009). Creencias sobre el rol de las tenologías de información y comunicación en la formación inicial de docentes: explorando las diferencias entre estudiantes y Docentes universitarios. Tesis Doctoral. Recuperado de: http://hdl.handle.net/2445/41486

Guerra, S., González, N., y García, R. (2010). Utilización de las TIC por el profesorado universitario como recurso didáctico. Revista Comunicar, 35(18), 141-148.

González, C. (2010). What do University Teachers Think eLearning is Good for in their Teaching? Studies in Higher Education, 35(1), 61-78.

Hattie, J. (2008). Visible Learning: A Synthesis of Over 800 Meta-Analyses Relating to Achievement. London. New York: Routledge.

Karsenti T., Meunier H., Raby C., y Villeneuve S., (2011). Usage des TIC en pédagogie universitaire : point de vue des étudiants. Ritpu, 8(3), 6-19.

Lebrun, M., Docq, F. y Smidts, D. (2008). Une plate-forme d'enseignement et d'apprentissage pour stimuler le développement pédagogique des enseignants et la qualité des enseignements: premières approches.Dans Actes du 25e colloque de l'AIPU. Montpellier, France: AIPU. Recuperado de http://www.aipu2008montpellier.fr

Maaroufi, F. (2016). Effets des TIC sur les pratiques pédagogiques dans un établissement d'enseignement supérieur marocain. Adjectif.net [En línea] http://www.adjectif.net/spip/spip.php?article385

Marton, F., y Pang, W. Y. (2005). On the Unit of Description in Phenomenography. Higher Education Research and Development, 24(4), 335-348.

Moersch, C. (1995). Levels of technology implementation (LoTi): A framework for measuring classroom technology use. Learning and Leading with Technology, 23(3), 40-42.

Peraya, D., y Peltier, C. (2012). Une année d'immersion dans un dispositif de formation aux technologies : prise de conscience du potentiel éducatif des TICE, intentions d'action et changement de pratique. Revue internationale des technologies en pédagogie universitaire, 9(1-2), 111-135. doi:10.7202/1012906ar. 